\title{
Dinâmica Industrial da Manufatura Brasileira: Análise Setorial de Padrões Persistentes
}

\author{
Ivette Luna (IE/UNICAMP) ${ }^{1}$ \\ Elias Youssef Haddad Netto (IE/UNICAMP) \\ Paulo Ricardo da Silva Oliveira (IE/UNICAMP)
}

\section{Resumo}

A heterogeneidade, a assimetria das distribuições e a persistência da posição relativa em relação às variáveis de desempenho e o caráter não persistente das taxas de crescimento das firmas têm sido alguns resultados empíricos verificados e amplamente discutidos no âmbito da microeconomia evolucionária. Estes fatos estilizados desafiam uma série de pressupostos adotados pelos modelos neoclássicos, como por exemplo, o artifício da firma representativa e a crença na eficiência do mercado enquanto mecanismo de seleção. Este artigo busca verificar se a heterogeneidade, assimetria, persistência das posições relativas e ausência de persistência nas taxas de crescimento ocorrem também para o caso da indústria brasileira. Para tanto, utilizase de métodos não-paramétricos, mais especificamente um conjunto de medidas descritivas agregadas e setoriais, de distribuições e matrizes de probabilidade de transição para dados no nível das firmas. Verificase a partir dos dados da Pesquisa Industrial Anual (PIA-IBGE), para o período de 1996-2013 e setores selecionados, a existência e persistência de heterogeneidade, assimetria e a alta probabilidade das firmas manterem suas posições relativas de desempenho no tempo, ao contrário do que ocorre com as taxas de crescimento. Os padrões observados são regulares e independem das especificidades setoriais e do corte temporal.

Palavras-chave: teoria evolucionária da firma, matrizes de transição, heterogeneidade das firmas.

\begin{abstract}
Firms' heterogeneity, asymmetric distribution, persistence of relative position in terms of performance variables and the unsteadiness of firms' growth rates are some of the empirical results found and broadly discussed within evolutionary economics. These stylized facts are challenging to several assumptions taken by mainstream economics, such as the idea of a representative firm and the trust in markets' ability to select most productive firms. In this paper, we seek to assess whether heterogeneity, asymmetry, persistence of relative positions and unsteadiness of growth rates occurs in the Brazilian industry as empirical evidence shows for other countries. For that, we employ non-parametric methods, more specifically a set of aggregate descriptive statistics, distributions and probability transition matrixes to infer about the occurrence of these stylized facts. Based on the Annual Industry Survey (PIA-IBGE) data for selected sectors from 1996-2013, we found high firm heterogeneity, high probabilities of firms keeping their relative positions in terms of performance and unsteadiness of growth rates across the years. Patterns are regular no matter sectorial specificities and period of analyses.
\end{abstract}

Keywords: evolutionary economics, transition matrices, firm heterogeneity.

Classificação JEL: L10, L22, L60.

\footnotetext{
${ }^{1}$ Autor principal. E-mail: $\underline{\text { iluna@unicamp.br }}$
} 


\section{Dinâmica Industrial Da Manufatura Brasileira: Análise Setorial De Padrões Persistentes}

\section{Introdução}

A crescente disponibilidade de dados longitudinais no nível das firmas tem possibilitado uma série de avanços para o tratamento de questões microeconômicas, sobretudo no campo da microeconomia evolucionária. No Brasil, os dados da Pesquisa Industrial Anual (PIA-IBGE) e da Pesquisa de Inovação (PINTEC - IBGE), intensificaram a realização de estudos empíricos sobre a indústria nacional que acompanham a fronteira das pesquisas internacionais, bem como o tratamento de novas questões, revelando e corroborando uma série de características da dinâmica industrial e da sua estrutura produtiva e organizacional, assim como os possíveis impactos no desenvolvimento econômico ${ }^{2}$. Estes estudos têm diferentes bases teóricas e nem sempre atingem consenso sobre os resultados e conclusões encontradas de forma empírica (De Negri e Cavalcante, 2013).

Dentre tais pesquisas, este trabalho destaca aquelas desenvolvidas no âmbito do arcabouço teórico evolucionário, que se fundamentam no levantamento rigoroso e robusto de evidências estatísticas que permitem melhor compreensão sobre as características e determinantes da dinâmica industrial (Catela, Cimoli e Porcile, 2015). A dinâmica industrial se dá sob constante mudança tecnológica, como pode ser observado a partir de uma série de padrões e indicadores, os quais são afetados pela própria dinâmica tecnológica (Dosi et al., 2008).

A literatura empírica, nesta frente de pesquisa, é vasta. Dentre as evidências coletadas, destaca-se a ubiquidade da heterogeneidade das firmas em termos de produtividade, tamanho, receita, lucro, dentre outros, independente do nível de agregação, de especificidades setoriais, períodos e recortes geográficos (Bartelsman e Doms, 2000; Syverson, 2011). Estes estudos destacam a natureza persistente e assimétrica dos indicadores citados e suas taxas de variação no tempo (Bottazzi et al., 2011; Bottazzi, Cefis e Dosi, 2002b; Dosi, 2005). A persistência é observada também em outras dimensões que caracterizam as firmas. Os trabalhos desenvolvidos por Cefis (2003) e Triguero e Córcoles (2013), por exemplo, mostram a persistência dos padrões de inovação no Reino Unido e Espanha, respetivamente.

Dosi et al. (2016) também apontam para a heterogeneidade das variáveis de desempenho das firmas e a assimetria das distribuições empíricas e distribuições com caldas pesadas para as taxas de crescimento. Destacam ainda, a persistência da turbulência nos mercados.

Por trás da heterogeneidade se observa a persistência nas posições relativas das firmas ao longo do tempo em termos de produtividade, receita, valor adicionado e outras variáveis que resultam de suas características intrínsecas. Evidentemente, taxas de crescimento que não persistem no tempo inviabilizam grandes mudanças na posição relativa das firmas. A persistência da heterogeneidade da firma no tempo desafia o pressuposto da racionalidade dos agentes maximizadores num contexto de informações perfeita e tecnologia como um bem público,

\footnotetext{
${ }^{2}$ No âmbito nacional, o uso de microdados nas pesquisas empíricas têm se intensificado nos últimos anos. Veja por exemplo o esforço do Instituto de Pesquisa Econômica Aplicada e da Associação Brasileira de Desenvolvimento Industrial, com a coletânea de trabalhos divulgados. (ver Messa, Alexandre; Romminger, Alfredo Eric; Auricchio Ledo et al., 2015)
} 
necessárias para que o mercado seja um bom mecanismo de seleção das firmas mais eficientes (Dosi et al., 2012). Este fato estilizado se confirma, sobretudo, em trabalhos importantes como Foster, Haltiwanger e Syverson (2008), Syverson (2011), Bartelsman e Dhrymes (1998), dentre outros.

Em relação às taxas de crescimento, seja das receitas, produtividades ou valores adicionados, tem-se verificado, ainda, que grandes episódios de crescimento ou contração são relativamente incomuns. De forma mais técnica, a distribuição do crescimento tem apresentado caldas relativamente longas para todos os setores industriais (Bottazzi et al., 2007; Dosi, 2005). Neste sentido, o catching-up e a destruição criadora schumpeteriana, parecem fenômenos incomuns a partir desta perspectiva (Dosi et al., 2012).

Por outro lado, a despeito da persistência da posição relativa das firmas, pode-se observar relevante turbulência nos mercados, através de fenômenos de entrada e saída e mudanças de parcelas de mercado (ver Baldwin e Rafiquzzaman, 1995; Bartelsman e Doms, 2000). Algumas análises apontam que entre $20 \%-40 \%$ dos entrantes saem do mercado nos dois primeiros anos de atividade, e somente 40-50\% sobrevivem mais de sete anos dado alguns contextos (Dosi, Pereira e Virgillito, 2016).

Alguns estudos, como o de Coad, Segarra e Teruel (2013) para firmas na Espanha, revelam que ao se controlar pela idade, a distribuição de tamanho das firmas pode se tornar menos assimétrica no tempo. Ou seja, embora firmas mais experientes geralmente detenham maiores parcelas de mercado e níveis superiores de receita e produtividade, a diminuição da assimetria é reflexo de menos variabilidade nas de taxas de crescimento, de lucratividade e de produtividade. Evidências empíricas também mostram que a variabilidade nas taxas de crescimento diminuem de forma inversamente proporcional ao tamanho das firmas - a chamada Lei de Escala (Scaling Law), embora no caso italiano esta relação não se observe nem mesmo para dados setoriais agregados a dois dígitos da ISIC (Bottazzi, Cefis e Dosi, 2002).

Estudos com matrizes de transição também apontam para a persistência da posição relativa das firmas e a não persistência das taxas de crescimento (Dosi et al., 2012; Dosi, Pereira e Virgillito, 2016; Eric J. Bartelsman, 1998; Mathew, 2017).

Este artigo visa contribuir com a caracterização da dinâmica industrial brasileira por meio da utilização de instrumentos não paramétricos de análise. Considera-se, explicitamente, que as firmas estão sujeitas a pressões adaptativas em resposta ao processo de seleção. De forma mais específica, o artigo busca identificar regularidades associadas aos fatos estilizados descritos através de medidas setoriais agregadas e matrizes de transição para as firmas de setores considerados da indústria de manufatura brasileira. A partir da literatura que fundamenta este trabalho, busca-se lançar luz, sobretudo, sobre as seguintes questões: 1) A heterogeneidade das firmas, bem como a assimetria da distribuição quando consideradas variáveis de desempenho das firmas dos diversos setores, são verificadas para o caso Brasileiro? 2) Há mudanças significativas na estrutura da manufatura, em relação aos níveis e às taxas de variação das variáveis de desempenho das firmas? 3) Existe persistência no tempo da posição relativa das firmas de um mesmo setor em relação aos níveis e taxas de variação das variáveis de desempenho?

A resposta a essas perguntas leva a um conhecimento mais aprofundado da estrutura produtiva nacional, e contribui para uma melhor compreensão dos determinantes da dinâmica industrial. Evidências adicionais sobre tais determinantes podem dar suporte ao processo de 
tomada de decisão dos formuladores de políticas, ja que explicitam processos de longo prazo, aparentemente atrelados a certa morfologia das firmas.

Este artigo está dividido em mais 3 seções além desta introdução. A seção 2 descreve o apresenta a metodologia e o banco de dados. A seção 3 traz os resultados. A secção 4, por fim, conclui o artigo.

\section{Bases de dados, proxies e método}

A pesquisa foi realizada a partir dos microdados da Pesquisa Industrial Anual para Empresas (PIA - Empresa). A PIA contém informações censitárias para as empresas brasileiras da indústria de manufatura e transformação com número de empregados maior ou igual a 30 estrato certo. A PIA abrange os anos entre 1996 e 2013, sendo a análise desenvolvida para a indústria de transformação em nível agregado e posteriormente desagregada por divisões a 2 dígitos (10 a 33) da Classificação Nacional de Atividade Econômica CNAE 2.0. É importante ressaltar que a amostra é responsável por pelo menos $80 \%$ do valor agregado total do setor de manufatura (Sebrae, 2014).

De forma a trabalhar com a maior quantidade de dados possíveis, a compatibilização das pesquisas de 1996 a 2007, que constam na base do IBGE ainda com a classificação da CNAE 1.0 foi realizada via tradutor oferecido pelo próprio IBGE (CONCLA/IBGE). As rotinas necessárias para a compatibilização das bases, deflacionamento das variáveis nominais e desenvolvimento dos exercícios empíricos foram implementadas no software $\mathrm{R}^{3}$. Os valores nominais foram transformados a preços de 2013 utilizando os deflatores setoriais construídos a partir das Contas Nacionais ${ }^{4}$, a 2 dígitos da CNAE 2.0.

Para a identificação das assimetrias, turbulência e persistência da heterogeneidade optouse por caracterizar às firmas a partir de um conjunto específico de variáveis: tamanho, produtividade do trabalho, ganhos de produtividade e crescimento das firmas ${ }^{5}$.

A produtividade do trabalho $\left(\pi_{i}\right)$ da $i$-ésima firma pode ser medida como a relação entre o valor adicionado (VA) ou a receita bruta por trabalhador ou por horas trabalhadas. Para os nossos propósitos, optamos por utilizar o valor da transformação industrial (VTI) por trabalhador como proxy para a produtividade do trabalho, uma vez que se busca analisar o impacto dos fatores internos às empresas na dinâmica industrial, apesar das várias críticas que esta medida pode sofrer, bem como o viés que a caracteriza pela exclusão de outros insumos na sua construção.

Apesar de ser possível estimar o VA para as empresas que participam da pesquisa, o VTI é uma proxy adequada para quantificar o valor adicionado. A diferença entre ambos os critérios, como indicado pelo IBGE, ocorre tanto nas receitas quanto nos custos considerados. O VTI considera no cálculo apenas os custos diretamente envolvidos na produção, tais como matérias-

${ }^{3}$ R Core Team (2015). R: A language and environment for statistical computing. R Foundation for Statistical Computing, Vienna, Austria. URL https://www.R-project.org/.

${ }^{4}$ Elaboração própria.

${ }^{5}$ Um conjunto extenso de variáveis ao nível das firmas foi observado, no entanto, não são apresentados neste trabalho por questões de espaço. Ainda deve ser notado que, os resultados aqui não colocados não diferem daqueles apresentados. 
primas, energia e manutenção, enquanto o VA também deduz outros tipos de despesas como publicidade, frete, entre outros. Para o cálculo do VTI, considera-se as receitas operacionais (sem as receitas financeiras) - isto é, resultantes dos produtos fabricados ou de variação no estoque. Ambos os conceitos são utilizados indistintamente pelo IBGE.

Com relação ao tamanho das firmas, utilizou-se o total de empregados e o total de receitas. Já a taxa de crescimento das firmas $\left(g_{i}\right)$ foi estimada pela diferença do logaritmo natural da receita total de um determinado período e do período anterior.

A análise não paramétrica, além da estatística descritiva regular, oferece instrumentos de estimação de distribuições empíricas, de análise multivariada e de análise dinâmica de sistemas por meio das matrizes de transição (Boldrini, 1986). Estes métodos têm se colocado como alternativa à análises paramétricas (Silverman, 1986). Assim, visando a análise geral da estrutura produtiva, aplicamos métodos não paramétricos a partir de: a) um conjunto de medidas descritivas agregadas e setoriais; b) um conjunto de distribuições e c) um conjunto de matrizes de transição de probabilidades.

Por meio da observação dos primeiros quatro momentos de variáveis-chave de desempenho, pode-se analisar a heterogeneidade estrutural independente do nível de desagregação e setor de atividade econômica. As assimetrias e as caldas pesadas das distribuições das variáveis de desempenho consideradas também podem ser observadas a partir das tabulações agregadas estimadas a partir das informações setoriais de toda a manufatura. Neste artigo, são apresentados e discutidos resultados para setores específicos, selecionados a partir da representatividade na amostra disponível e relevância para a economia brasileira em termos de participação do setor no PIB. Os setores selecionados foram Fabricação de Produtos alimentícios (CNAE - 10), Fabricação de Produtos Químicos (CNAE-20), Fabricação de Máquinas e Equipamentos (CNAE -28) e Fabricação de Veículos Automotores (CNAE-29).

Potr outro lado, as distribuições persistentes ao longo do tempo sugerem persistência da heterogeneidade estrutural que também se observa nos seus momentos e principalmente nos seus coeficientes de assimetria e curtose, significativamente diferentes das observadas em uma distribuição normal (Bottazzi, Cefis e Dosi, 2002a). Ainda, as caldas pesadas mostram um convívio entre firmas com baixo e alto desempenho. De fato, as assimetrias nas distribuições de produtividade, tamanho e crescimento setoriais apontam que as firmas se afastam das médias observadas, enfatizando a diversidade do mercado. Ainda, sendo esta assimetria positiva, implicam em aumento na frequência de observações à esquerda da media observada.

Ainda, dada a heterogeneidade observada no desempenho das firmas nos diversos setores, as matrizes de probabilidades de transição, ou simplesmente matrizes de transição (MT) são instrumentos da estatística não paramétrica e da economia matemática que nos permitem analisar o desempenho das firmas no tempo. Estas são baseadas em modelos markovianos, ou seja, considera-se o sistema dinâmico como um processo estocástico em que a probabilidade de um sistema estar em um estado $j$ no período $t+1$ depende somente do estado $i$ em que o sistema esteve no período $t$. Assim, trata-se de uma análise de curto prazo.

As probabilidades de transição são agrupadas na matriz de probabilidades transição $p_{i j}$, com $i, j=1, \ldots, n$, onde o primeiro subscrito associa-se ao período inicial ou atual e o segundo subscrito indexa o próximo período. Assim, $p_{i j} \geq 0$ é a probabilidade de que o sistema estará no estado $j$ no próximo período, dado que o estado atual é $i$ (Mathew, 2017). Portanto, a soma dos 
$p_{i j}$ 's sobre $i$ - ou seja, a soma dos elementos de cada coluna - deve ser igual à unidade. Considerase ainda que as probabilidades $p_{i j}$ são fixas e independentes de $t$.

Neste trabalho, os estados são dados pelos quintis equipopulados de tamanho (receita total), produtividade $\left(\pi_{i}\right)$, de variações de produtividade $\left(\Delta \pi_{i}\right)$ e de crescimento $\left(g_{i}\right)$, respectivamente, tendo assim um total de três conjuntos de MTs. Com estas matrizes será possível observar alguns aspectos da dinâmica industrial, principalmente a persistência dos estados e a turbulência representada pelos elementos fora da diagonal principal das matrizes, que indicam alterações nos estados entre períodos. Quanto maior as probabilidades estimadas na diagonal principal das matrizes, maior a persistência no desempenho das firmas. Deve-se destacar também que o conceito de persistência aqui utilizado deve ser entendido como a probabilidade de a firma manter o seu desempenho observado no período anterior ou inicial, sem excluir a turbulência intragrupo (quintil).

\section{Resultados}

\subsection{Análise das estatísticas descritivas e das distribuições}

Nesta seção apresentamos os resultados descritivos e a análise das distribuições. Para as estatísticas descritivas, foram calculados, inicialmente, as medianas, desvios padrão e coeficientes de curtose e de assimetria em relação ao número de firmas, receitas, produtividade e respectivas taxas de variação. Posteriormente, foram calculados os valores médios para o período (1996-2005), a partir das médias anuais calculadas previamente. A Tabela 1 apresenta as estatísticas do período para os setores selecionados. 
Tabela 1. Estatísticas Descritivas de setores selecionados para o período de 1996-2013

\begin{tabular}{|c|c|c|c|c|c|c|c|c|c|c|}
\hline \multirow[t]{2}{*}{ Setor } & \multirow{2}{*}{$\begin{array}{c}\text { Metricas sobre Amostras } \\
1996-2013\end{array}$} & \multirow{2}{*}{$\#$} & \multicolumn{4}{|c|}{ Receita Total* } & \multicolumn{4}{|c|}{ Produtividade* } \\
\hline & & & Med & DP & Assim. & Curt. & Med & DP & Assim. & Curt. \\
\hline \multirow{2}{*}{10} & Media & 2,943 & 136,980 & 784,609 & 17.1 & 374.1 & 88.9 & 154.6 & 9.0 & $\overline{182.4}$ \\
\hline & Mediana & 2,854 & 136,193 & 816,448 & 17.9 & 393.1 & 78.5 & 134.5 & 8.1 & 132.8 \\
\hline \multirow{2}{*}{20} & Media & 1,132 & 186,359 & 768,433 & 12.1 & 222.4 & 190.4 & 281.3 & 5.4 & 63.6 \\
\hline & Mediana & 1,184 & 179,028 & 756,897 & 11.4 & 175.4 & 170.8 & 277.6 & 5.6 & 56.0 \\
\hline \multirow{2}{*}{28} & Media & 1,710 & 55,093 & 226,131 & 12.7 & 225.5 & 107.2 & 130.8 & 7.8 & 157.5 \\
\hline & Mediana & 1,662 & 54,902 & 237,760 & 13.3 & 228.6 & 103.0 & 113.0 & 4.6 & 45.2 \\
\hline \multirow{2}{*}{29} & Media & 917 & 245,552 & $1,622,609$ & 12.9 & 192.1 & 91.0 & 117.4 & 8.1 & 137.2 \\
\hline & Mediana & 921 & 240,341 & $1,609,277$ & 12.9 & 191.1 & 88.9 & 107.8 & 6.3 & 80.9 \\
\hline \multirow{4}{*}{ Total Manuf. } & Media & 1,059 & 160,037 & $1,017,570$ & 11.6 & 198.8 & 95.6 & 155.5 & 6.8 & 108.8 \\
\hline & Mediana & 829 & 68,239 & 263,743 & 11.3 & 155.8 & 80.7 & 103.9 & 5.7 & 52.9 \\
\hline & Max & 4,387 & $1,709,308$ & $19,104,451$ & 29.4 & $1,111.2$ & 550.1 & $1,355.3$ & 49.3 & 2,901 \\
\hline & Min & 26 & 7,017 & 27,269 & 3.4 & 15.8 & 22.4 & 29.8 & 0.4 & 2.0 \\
\hline \multirow[t]{2}{*}{ Setor } & \multirow{2}{*}{$\begin{array}{c}\text { Metricas sobre Amostras } \\
1996-2013\end{array}$} & & \multicolumn{4}{|l|}{ Crescimento } & \multicolumn{4}{|c|}{ Var. Produtividade } \\
\hline & & & Med & DP & Assim. & Curt. & Med & DP & Assim. & Curt. \\
\hline \multirow{3}{*}{10} & Media & & $3.5 \%$ & $44 \%$ & $(0.06)$ & 21.3 & $-0.5 \%$ & $158 \%$ & $(0.19)$ & 44.8 \\
\hline & Media Geometrica & & $3.4 \%$ & & & & $-0.7 \%$ & & & \\
\hline & Mediana & & $4.2 \%$ & $43 \%$ & $(0.03)$ & 20.2 & $-1.3 \%$ & $149 \%$ & $(0.57)$ & 43.4 \\
\hline \multirow{3}{*}{20} & Media & & $2.7 \%$ & $36 \%$ & $(0.07)$ & 18.6 & $-1.0 \%$ & $94 \%$ & 0.18 & 45.7 \\
\hline & Media Geometrica & & $2.6 \%$ & & & & $-1.2 \%$ & & & \\
\hline & Mediana & & $3.4 \%$ & $36 \%$ & 0.01 & 17.3 & $0.3 \%$ & $96 \%$ & $(0.65)$ & 37.6 \\
\hline \multirow{3}{*}{28} & Media & & $1.7 \%$ & $39 \%$ & $(0.16)$ & 13.2 & $-1.7 \%$ & $83 \%$ & $(0.51)$ & 54.2 \\
\hline & Media Geometrica & & $1.3 \%$ & & & & $-1.9 \%$ & & & \\
\hline & Mediana & & $0.8 \%$ & $39 \%$ & 0.03 & 11.6 & $-2.9 \%$ & $79 \%$ & $(0.19)$ & 34.2 \\
\hline \multirow{3}{*}{29} & Media & & $2.3 \%$ & $36 \%$ & 0.29 & 18.6 & $-0.4 \%$ & $100 \%$ & 0.66 & 51.3 \\
\hline & Media Geometrica & & $1.9 \%$ & & & & $-0.6 \%$ & & & \\
\hline & Mediana & & $1.3 \%$ & $36 \%$ & 0.21 & 13.3 & $1.9 \%$ & $93 \%$ & 0.15 & 36.0 \\
\hline \multirow{4}{*}{ Total Manuf. } & Media & & $2.6 \%$ & $42 \%$ & 0.10 & 18.6 & $-0.5 \%$ & $111 \%$ & $(0.10)$ & 39.4 \\
\hline & Mediana & & $2.9 \%$ & $40 \%$ & 0.08 & 13.1 & $0.0 \%$ & $99 \%$ & $(0.15)$ & 32.5 \\
\hline & Max & & $41.0 \%$ & $96 \%$ & 12.79 & 241.8 & $39.6 \%$ & $286 \%$ & 8.04 & 142.9 \\
\hline & Min & & $-101.9 \%$ & $27 \%$ & $(10.48)$ & 2.6 & $-90.6 \%$ & $49 \%$ & $(9.06)$ & 3.1 \\
\hline
\end{tabular}

Código dos setores: Fabricação de Produtos alimentícios (CNAE - 10), Fabricação de Produtos Químicos (CNAE-20), Fabricação de Máquinas e Equipamentos (CNAE -28) e Fabricação de Veículos Automotores (CNAE-29). \# para o número de firmas do setor. Total da Manufatura considera dados empilhados para todos os anos dos setores 10-33.

*Valores em BRL K.

Fonte: Elaboração própria.

Observa-se, de maneira mais geral, que todos os setores analisados lograram taxas de crescimento de longo prazo positivas (média geométrica), sendo a maior para o setor de fabricação de alimentos (CNAE-10), 3,4\%, e a menor para fabricação de máquinas e equipamentos (CNAE28), 1,3\%.Nota-se, que os padrões de assimetria e dispersão são distintos quando considera-se os níveis e as taxas de variação, como também verificado em outros trabalhos empíricos internacionais (ver Dosi et al. , 2016). Os padrões de assimetria são muito menores para as taxas de crescimento, embora a dispersão se mantenha em níveis elevados.

Considerando-se o tamanho, medido pelas receitas, e a produtividade das firmas nos diferentes setores, nota-se que a elevada dispersão verificadas pelos desvios-padrões e distribuições bastante leptocúrticas, corroboram a evidente ubiquidade da heterogeneidade das firmas, como também verificado em outros estudos empíricos para outros países (ver Bartelsman e Doms, 2000; Syverson, 2011). A elevada assimetria positiva revela a exacerbada tolerância à coexistência de um número elevado de firmas com baixa produtivade e poucas firmas na fronteira 
tecnológica, fenômeno identificado como neo-dualismo ${ }^{6}$ na literatura recente. Este fato estilizado coloca dúvidas sobre a eficiência dos mercados enquanto mecanismo de seleção das firmas mais eficientes.

Em relação ao tamanho, as medidas de dispersão e assimetria apontam, também, para elevada heterogeneidade e a coexistência de um grande número de firmas pequenas e poucas firmas de grande porte. Este fato estilizado desafia alguns pressupostos da teoria da produção e dos custos, no que tange a existência de escalas ótimas de eficiência. Se a escala ótima de eficiência e economias de escalas fossem fatores limitantes ou centrais para explicar o tamanho das firmas, seria razoável supor níveis menos exacerbados de heterogeneidade e assimetria das firmas.

Em relação às taxas de crescimento das firmas e a variação da produtividade, assimetrias próximas de zero indicam proporção equilibrada entre as firmas de crescimento e ganhos de produtividade a cima e abaixo da média da indústria. Embora as distribuições ainda sejam, na média, leptocúrticas, com caldas consideravelmente longas, esses valores são bem menos expressivos quando comparados com a curtose da distribuição das receitas e da produtividade. Em outras palavras a probabilidade de encontrar valores extremos é menor. Os desvios padrões elevados, indicam a forte heterogeneidade das firmas em relação as taxas de crescimento e variação da produtividade.

As últimas linhas da Tabela 1, revelam que quando considerado o total da manufatura a heterogeneidade e dispersão ainda são verificadas para as receitas, produtividade e respetivas taxas de variação. No entanto, os valores mínimos indicam a existência de setores com baixa assimetria e dispersão, bem como de oligopólios concentrados.

Verificada a heterogeneidade e assimetria da indústria, é preciso analisar se esse padrão é persistente no tempo, tanto para os níveis como para as taxas de variação. A sequência de gráficos a seguir apresenta os primeiros momentos - média, desvio padrão, assimetrias e curtoses - para as receitas totais, produtividade, taxas de crescimento e variação da produtividade entre 1996-2013 para os quatro setores selecionados: Fabricação de Produtos Alimentícios (CNAE - 10), Fabricação

${ }^{6} \mathrm{O}$ termo dualismo tem sido utilizado historicamente para descrever a existência simultânea de setores modernos e tradicionais, sob a hipótese de que processo de industrialização promoveria a expansão de setores modernos e a supressão de setores atrasados ou tradicionais. 
de Produtos Químicos (CNAE-20), Fabricação de Máquinas e Equipamentos (CNAE -28) e Fabricação de Veículos Automotores (CNAE-29).
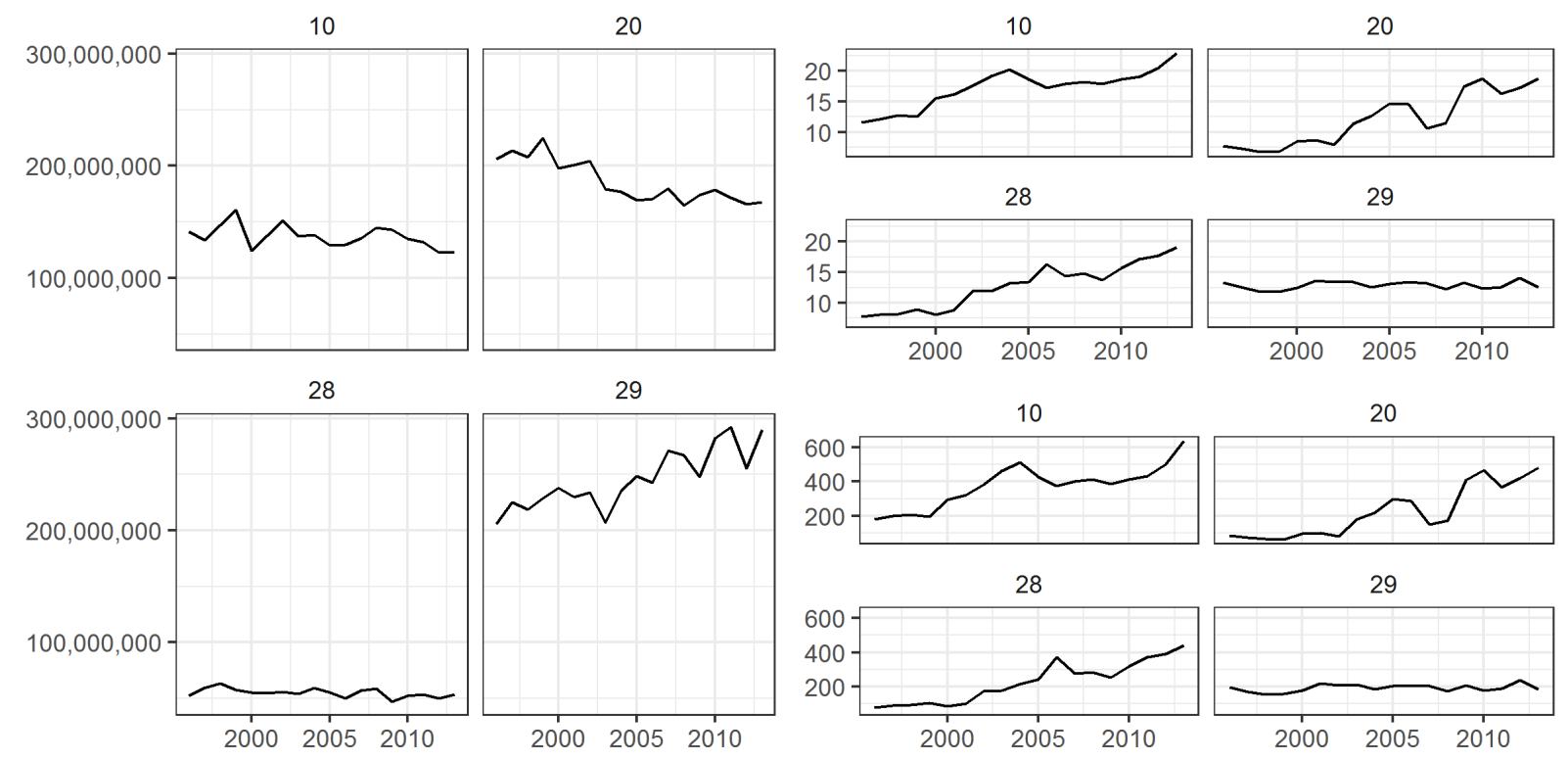

Figura 1. Três momentos da Receita ao nível das firmas para o período de 1996 a 2013 nos setores selecionados: Esquerda) Média; Direita Superior) assimetria; Direita inferior) curtose ${ }^{7}$.

Fonte: Elaboração própria.

Na Figura 1 observa-se, inicialmente, tendência de quedas nas receitas totais médias ao longo da série para todos os setores, com exceção do setor de fabricação de veículos automotores (29). As receitas totais médias, dos setores que tiveram decrescimento, variaram entre - 10 e - $20 \%$ quando considerada a média do triênio 1996-98 vs 2011-13, enquanto o setor de veículos automotores cresceu $29 \%$ no mesmo período. A distribuição das receitas deste setor é a mais estável da série quando observamos a variação da assimetria e da curtose, indicando que o setor sofreu menos alterações estruturais em relação ao tamanho das firmas - 4\% na assimetria e 30\% na curtose, considerando-se a média dos três anos iniciais e finais. Os demais setores, no entanto, apresentam forte aumento das curtoses e assimetrias para o mesmo período de comparação - o setor de produtos químicos (20) teve um crescimento de $140 \%$ na assimetria e $484 \%$ na curtose e uma taxa de decrescimento das receitas de $20 \%$.

Contudo, todos os setores apresentaram aumentos significativos da assimetria positiva e do peso das caldas no período analisado - sempre considerando a comparação entre a média dos três primeiros e três últimos anos da série. Esse resultado se verifica para todos os setores independente de crescimento ou decrescimento das receitas. Porém, o aumento da assimetria positiva foi mais acentuado para os 3 setores que apresentaram decrescimento. Em outras palavras, receitas médias decrescentes coincidem com maior proporção de firmas relativamente menores na distribuição destes setores - isto é, com menores capacidades de geração de receitas. Já para o setor 29,

${ }^{7} \mathrm{O}$ desvio padrão para as receitas totais em níveis retornou valores bastante altos. Dada a disparidade de escalas, optou-se por não plotar os desvios-padrão na Figura 3. 
verificou-se aumento pouco expressivo da assimetria positiva e tolerância setorial (visto pelas elevadas curtoses).

A Figura 2 apresenta os mesmos momentos para a produtividade setorial.

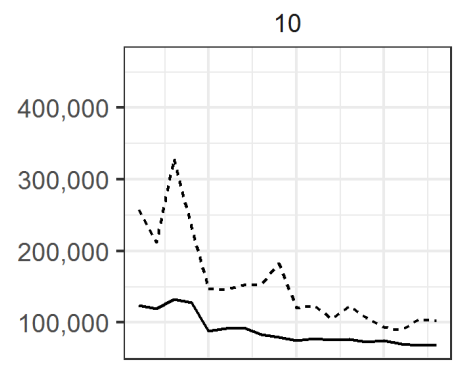

28

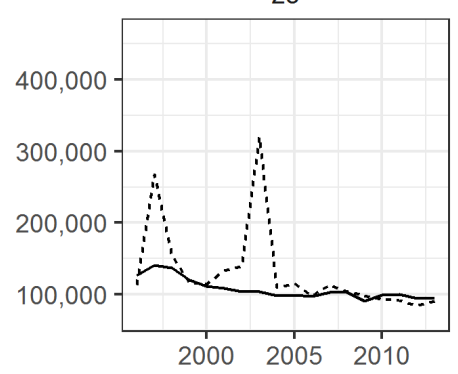

20

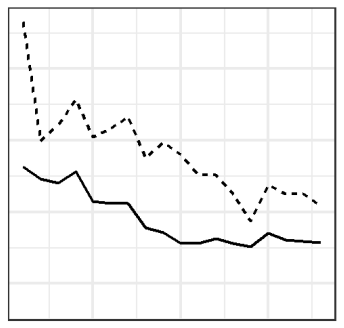

29

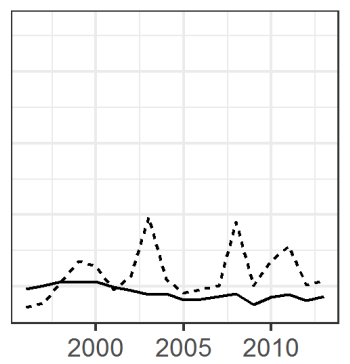

10

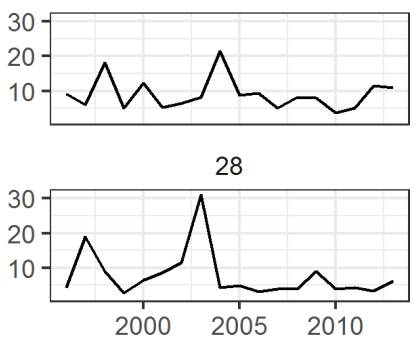

10

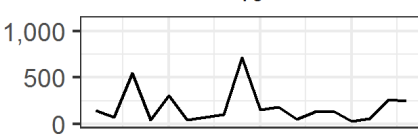

28

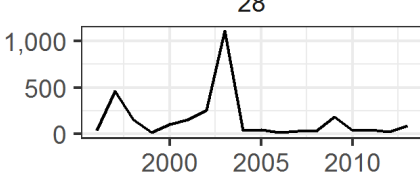

20

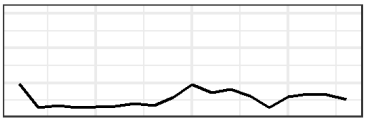

29

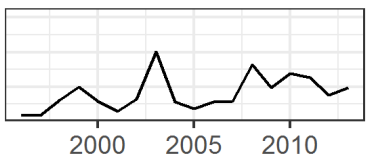

20

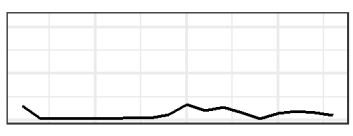

29

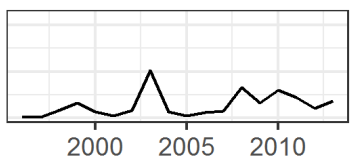

Figura 2. Os primeiros quatro momentos da Produtividade ao nível das firmas para o período de 1996 a 2013 nos setores selecionados: Esquerda) Média (continua) e Desvio-padrão (pontilhado); Direita Superior) assimetria; Direita inferior) curtose.

Nota-se, para a produtividade, uma queda para os quatro setores, ainda que em proporções distintas. Novamente, o setor de veículos automotores (29) é o que apresenta menor queda quando se compara o resultado dos três primeiros e três últimos anos da série (-16\%). O setor de fabricação de produtos alimentícios (10) e o setor de fabricação de produtos químicos (20) foram os que tiveram maiores reduções da produtividade do trabalho, $(-45 \%)$ e $(-36 \%)$ respectivamente. As assimetrias e tolerância setorial, no entanto, aumentaram mais expressivamente para o setor que obteve menor decrescimento da produtividade, isto é, o setor de veículos automotores. Isto significa que a proporção de firmas menos produtivas aumentou (dado o aumento da assimetria de $211 \%$ ) e a tolerância setorial com firmas menos produtivas também (aumento da curtose em $445 \%$ ). Ainda assim, a produtividade do setor caiu menos, relativamente aos demais setores considerados. Esta relação entre menores decrescimentos da produtividade dos setores e maiores assimetrias e curtoses parece robusta para os setores considerados. Os setores que decresceram mais em termos de produtividade obtiveram aumentos menos expressivos da assimetria e da tolerância setorial - alimentos (assimetria $-17 \%$ e curtose -26\%) e químicos (assimetria 14\% e curtose $21 \%$ ).

É importante ressaltar que os dados revelam alta correlação positiva entre as curtoses e assimetrias para todos os setores e variáveis em níveis consideradas na análise. O formato quase idêntico da curtose e da assimetria nas figuras 3 e 4 confirmam tal correlação. Embora esse fato deva ser melhor estudado, podemos afirmar que setores que sofreram maiores assimetrias positivas - aumento da proporção de firmas menores ou de firmas menos produtivas - também aumentaram a tolerância setorial em relação a tamanho ou produtividade. 
A Figura 3 e 4 apresentam os momentos para a taxa de crescimento, ou variação das receitas, e variação da produtividade.
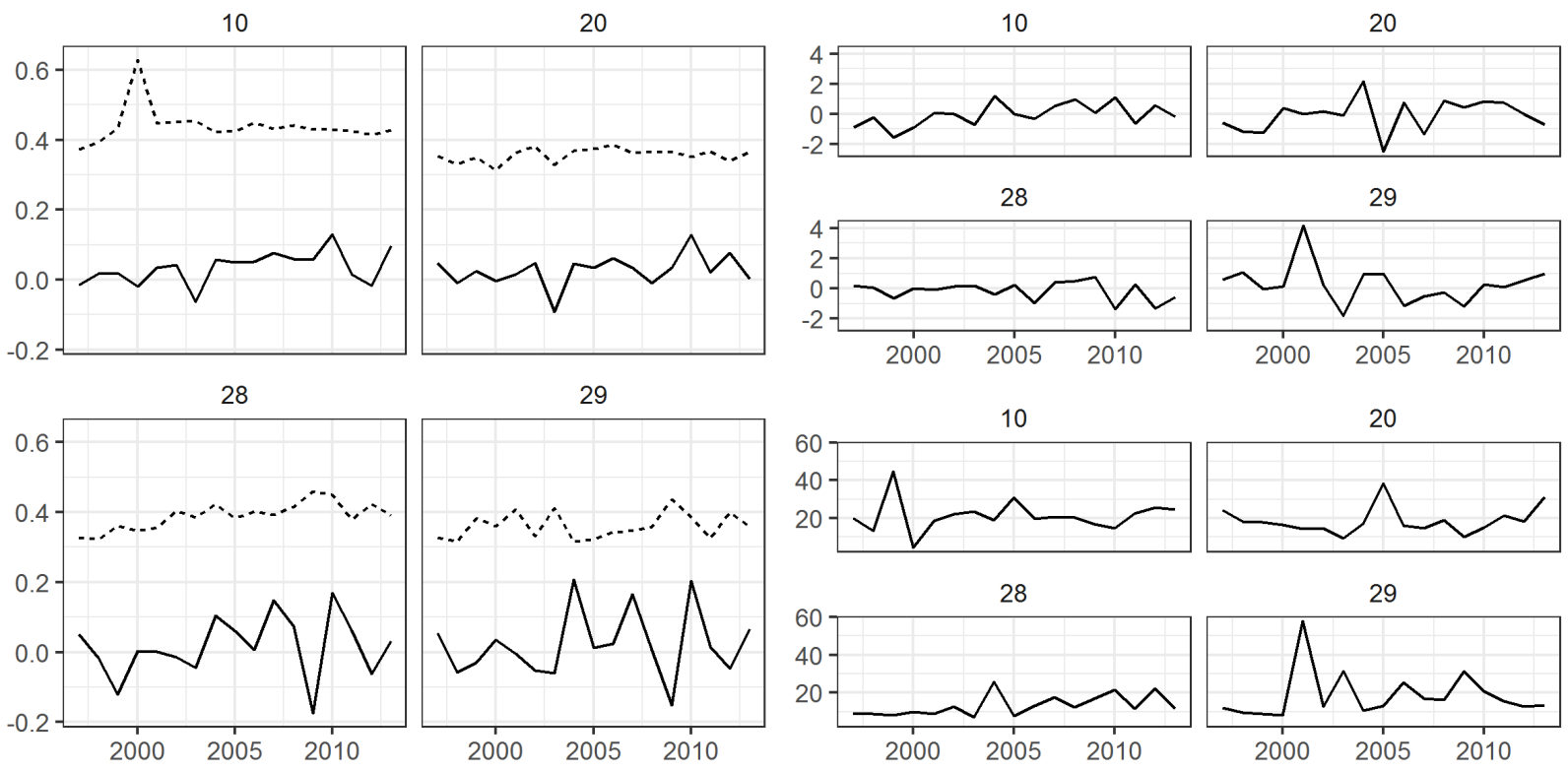

Figura 3. Os primeiros quatro momentos da Taxa de Crescimento ao nível das firmas para o período de 1996 a 2013 nos setores selecionados: Esquerda) Média (continua) e Desvio-padrão (pontilhado); Direita Superior) assimetria; Direita inferior) curtose.

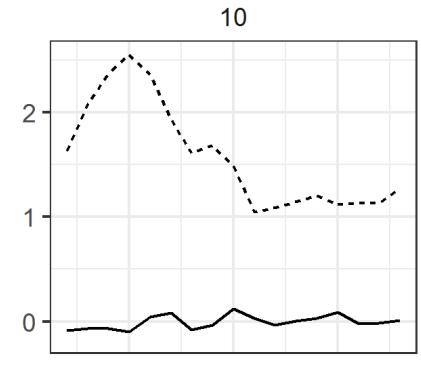

28

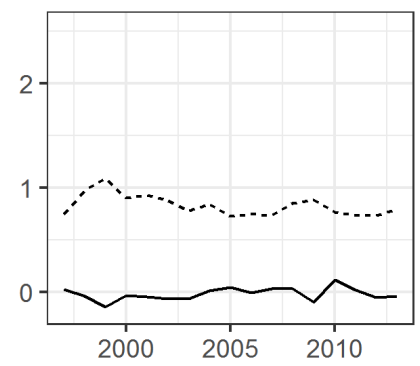

20

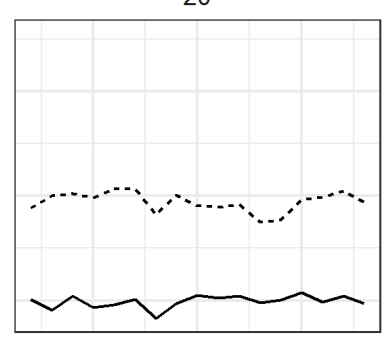

29

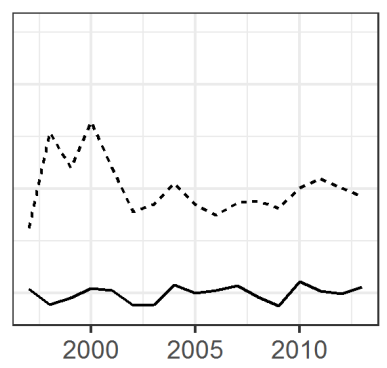

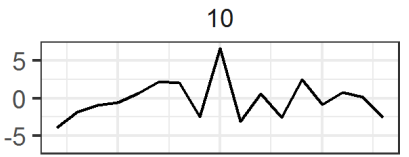

28

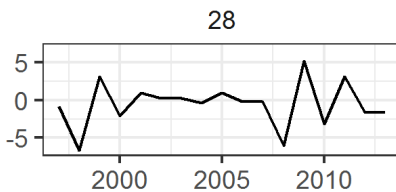

10

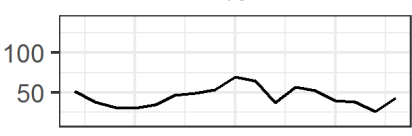

28

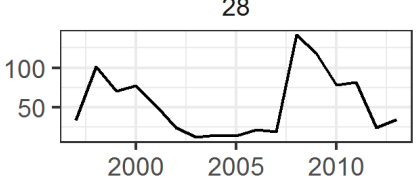

20
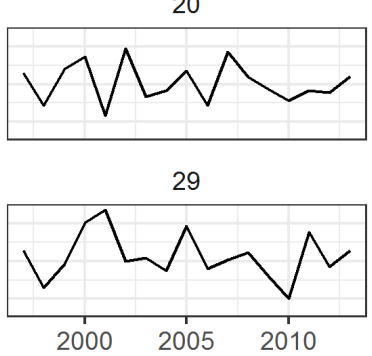

20

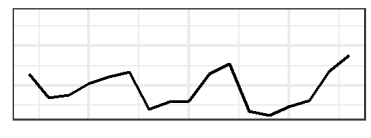

29

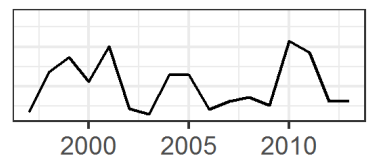

Figura 4. Os primeiros quatro momentos da Variação da Produtividade ao nível das firmas para o período de 1996 a 2013 nos setores selecionados: Esquerda) Média (continua) e Desvio-padrão (pontilhado); Direita Superior) assimetria; Direita inferior) curtose.

Como esperado, tanto comportamento da taxa de crescimento das firmas como a variação da produtividade no tempo apresentam padrões distintos das variáveis em níveis. As assimetrias e 
as curtoses se mantém em patamares muito mais baixos, indicando caldas menos pesadas e baixas assimetrias positivas e negativas. As taxas de crescimento do setor apresentam evidente instabilidade, mas sem grandes mudanças de patamares - em conformidade com o padrão revelado pelas médias na tabela 1 e resultados de estudos internacionais sobre o crescimento das firmas (ver Mathew, 2013)

Em suma, as distribuições persistentes ao longo do tempo corroboram a persistência da heterogeneidade estrutural que também se observa nos seus momentos e principalmente nos seus coeficientes de assimetria e curtose, significativamente diferentes das observadas em uma distribuição normal, como também verificado em estudos internacionais. Ainda, as caldas pesadas mostram um convívio entre firmas com baixo e alto desempenho, o que, em certa medida, questiona o papel do mercado como um bom mecanismo de seleção nos moldes neoclássicos. Ainda, sendo esta assimetria positiva, implicam em aumento na frequência de observações à esquerda da media observada, isto é, da existência de firmas consideradas menos produtivas ou menores.

\subsection{Matrizes de Transição}

Nesta seção apresentamos os resultados das matrizes de transição (MT). A Tabela 2 traz as MTs para a receita total, considerando-se as médias anuais das receitas das firmas e dos desviospadrão. Como descrito na metodologia, foram considerados 5 quintis de produtividade na composição das matrizes. A análise das matrizes permite verificar a tendência de persistência da posição relativa das firmas, de maneira a produzir informações complementares em relação a análise dos dados setoriais da seção anterior.

Tabela 2. Matrizes de transição para a Receita Total. Medias e Desvios-padrão das probabilidades de transição observadas para os quatro setores selecionados de 1996-2013.

10

\begin{tabular}{|c|c|c|c|c|c|}
\hline & \multicolumn{5}{|c|}{$t+1$} \\
\hline & 1 & 2 & 3 & 4 & 5 \\
\hline \multirow{2}{*}{1} & 88 & 16 & 1 & 0 & 0 \\
\hline & (2.1) & (3.3) & (0.4) & $(0.2)$ & $(0.1)$ \\
\hline \multirow{2}{*}{2} & 11 & 75 & 14 & 1 & 0 \\
\hline & (2.1) & (3.8) & (4.5) & $(0.3)$ & (0.1) \\
\hline \multirow{2}{*}{3} & 1 & 8 & 77 & 14 & 0 \\
\hline & $(0.3)$ & (3.0) & (6.7) & (4.3) & $(0.2)$ \\
\hline \multirow{2}{*}{4} & 0 & 1 & 8 & 80 & 9 \\
\hline & $(0.2)$ & $(0.6)$ & (2.8) & (6.3) & (2.8) \\
\hline \multirow{2}{*}{5} & 0 & 0 & 0 & 5 & 90 \\
\hline & $(0.0)$ & $(0.1)$ & $(0.3)$ & $(2.7)$ & (2.8) \\
\hline
\end{tabular}

28

28

\begin{tabular}{ccccccc}
\multicolumn{1}{c}{} & \multicolumn{1}{c}{1} & 2 & 3 & 4 & 5 \\
\cline { 3 - 8 } & $\mathbf{1}$ & $\mathbf{8 6}$ & $\mathbf{1 3}$ & $\mathbf{1}$ & $\mathbf{0}$ & $\mathbf{0}$ \\
& $\mathbf{4}$ & $(4.2)$ & $(4.0)$ & $(0.6)$ & $(0.2)$ & $(0.1)$ \\
& $\mathbf{1 2}$ & $\mathbf{7 0}$ & $\mathbf{1 8}$ & $\mathbf{1}$ & $\mathbf{0}$ \\
& $\mathbf{1}$ & $(4.1)$ & $(3.2)$ & $(3.0)$ & $(0.7)$ & $(0.1)$ \\
& $\mathbf{3}$ & $\mathbf{1}$ & $\mathbf{1 5}$ & $\mathbf{6 8}$ & $\mathbf{1 7}$ & $\mathbf{0}$ \\
& $\mathbf{3}$ & $(0.7)$ & $(4.1)$ & $(2.8)$ & $(3.3)$ & $(0.3)$ \\
& $\mathbf{4}$ & $\mathbf{0}$ & $\mathbf{2}$ & $\mathbf{1 3}$ & $\mathbf{7 3}$ & $\mathbf{1 1}$ \\
& $\mathbf{4}$ & $(0.4)$ & $(1.1)$ & $(3.1)$ & $(2.6)$ & $(2.2)$ \\
& & $\mathbf{0}$ & $\mathbf{0}$ & $\mathbf{0}$ & $\mathbf{8}$ & $\mathbf{8 9}$ \\
& $\mathbf{5}$ & $(0.0)$ & $(0.2)$ & $(0.3)$ & $(2.4)$ & $(2.2)$
\end{tabular}

20

\begin{tabular}{l|ccccc}
\multicolumn{1}{c}{} & \multicolumn{5}{c}{$\mathbf{t + 1}$} \\
$\mathbf{1}$ & $\mathbf{2}$ & $\mathbf{3}$ & $\mathbf{4}$ & $\mathbf{5}$ \\
\cline { 2 - 6 } $\mathbf{1}$ & $\mathbf{9 0}$ & $\mathbf{1 0}$ & $\mathbf{0}$ & $\mathbf{0}$ & $\mathbf{0}$ \\
& $(4.0)$ & $(3.8)$ & $(0.4)$ & $(0.3)$ & $(0.1)$ \\
$\mathbf{2}$ & $\mathbf{9}$ & $\mathbf{7 9}$ & $\mathbf{1 5}$ & $\mathbf{0}$ & $\mathbf{0}$ \\
& $(4.0)$ & $(3.5)$ & $(3.6)$ & $(0.3)$ & $(0.1)$ \\
$\mathbf{3}$ & $\mathbf{0}$ & $\mathbf{1 0}$ & $\mathbf{7 7}$ & $\mathbf{1 3}$ & $\mathbf{0}$ \\
& $(0.5)$ & $(3.4)$ & $(2.3)$ & $(2.7)$ & $(0.2)$ \\
$\mathbf{4}$ & $\mathbf{0}$ & $\mathbf{0}$ & $\mathbf{7}$ & $\mathbf{8 2}$ & $\mathbf{8}$ \\
& $(0.3)$ & $(0.5)$ & $(1.9)$ & $(2.3)$ & $(1.9)$ \\
$\mathbf{5}$ & $\mathbf{0}$ & $\mathbf{0}$ & $\mathbf{0}$ & $\mathbf{4}$ & $\mathbf{9 2}$ \\
& $(0.1)$ & $(0.2)$ & $(0.3)$ & $(1.7)$ & $(1.8)$
\end{tabular}

29

\begin{tabular}{|c|c|c|c|c|}
\hline & \multicolumn{4}{|c|}{$t+1$} \\
\hline 1 & 2 & 3 & 4 & 5 \\
\hline 86 & 14 & 1 & 0 & 0 \\
\hline (5.3) & $(4.0)$ & $(0.7)$ & $(0.2)$ & $(0.0)$ \\
\hline 13 & 73 & 12 & 0 & 0 \\
\hline (5.2) & (3.3) & (3.9) & $(0.5)$ & $(0.2)$ \\
\hline 1 & 12 & 77 & 10 & 0 \\
\hline (0.7) & (4.9) & $(4.6)$ & (3.0) & $(0.4)$ \\
\hline 0 & 0 & 10 & 83 & 7 \\
\hline$(0.3)$ & $(0.6)$ & (5.3) & (2.5) & (2.8) \\
\hline 0 & 0 & 0 & 6 & 93 \\
\hline$(0.0)$ & $(0.1)$ & $(0.0)$ & (3.2) & (2.7) \\
\hline
\end{tabular}


Como verificado por estudos para outros países, a probabilidade de uma firma manter sua posição relativa em termos de receitas do período $t$ para o período $t+1$ é bastante alta - como pode ser visto pela concentração das maiores probabilidades $(>68 \%$ ) na diagonal principal da matriz, para todos os setores considerados. Em outras palavras, as firmas raramente mudam de quintil de tamanho de um ano para o outro, e quando o fazem, logram, frequentemente, posições no quintil imediatamente superior ou inferior. Estudos que agregam mais anos para diminuir a sazonalidade e permitir períodos mais longos de mudança de tamanho das firmas encontraram probabilidades ligeiramente maiores fora da diagonal das matrizes, mas corroboraram a persistência das posições relativas de tamanho (Dosi et al., 2012). A Tabela 3 apresenta a mesma análise para a produtividade.

Tabela 3. Matrizes de transição para a Produtividade (VTI/ PO). Medias e Desvios-padrão das probabilidades de transição observadas para os quatro setores selecionados de 1996-2013.

10

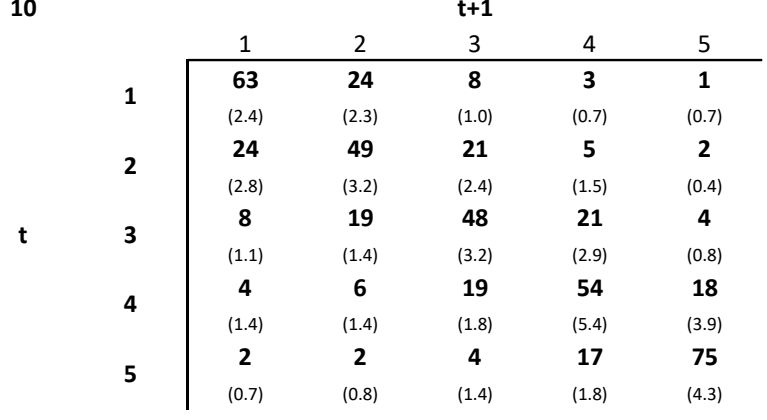

28

\begin{tabular}{ccccccc} 
& & 1 & 2 & 3 & 4 & 5 \\
\cline { 3 - 7 } & $\mathbf{1}$ & $\mathbf{6 6}$ & $\mathbf{2 2}$ & $\mathbf{6}$ & $\mathbf{2}$ & $\mathbf{2}$ \\
& $\mathbf{2}$ & $(2.8)$ & $(2.8)$ & $(1.0)$ & $(1.1)$ & $(0.6)$ \\
$\mathbf{t}$ & $\mathbf{2 2}$ & $\mathbf{4 7}$ & $\mathbf{2 1}$ & $\mathbf{7}$ & $\mathbf{2}$ \\
& $\mathbf{3}$ & $(1.4)$ & $(2.5)$ & $(3.0)$ & $(2.1)$ & $(0.8)$ \\
& $\mathbf{6}$ & $\mathbf{2 3}$ & $\mathbf{4 5}$ & $\mathbf{2 2}$ & $\mathbf{5}$ \\
& $\mathbf{4}$ & $(1.5)$ & $(1.8)$ & $(3.0)$ & $(2.8)$ & $(1.6)$ \\
& $\mathbf{3}$ & $\mathbf{6}$ & $\mathbf{2 2}$ & $\mathbf{5 1}$ & $\mathbf{1 8}$ \\
& $\mathbf{5}$ & $(1.2)$ & $(1.5)$ & $(2.4)$ & $(3.9)$ & $(1.8)$ \\
& $\mathbf{2}$ & $\mathbf{2}$ & $\mathbf{5}$ & $\mathbf{1 8}$ & $\mathbf{7 3}$ \\
& $(1.4)$ & $(0.9)$ & $(1.9)$ & $(1.8)$ & $(2.7)$
\end{tabular}

20

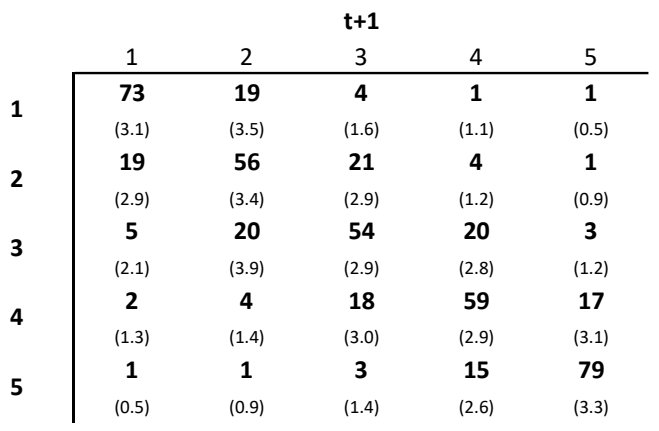

29

\begin{tabular}{cc|ccccc} 
& & 1 & 2 & 3 & 4 & 5 \\
\cline { 3 - 7 } & $\mathbf{1}$ & $\mathbf{6 6}$ & $\mathbf{2 3}$ & $\mathbf{5}$ & $\mathbf{2}$ & $\mathbf{2}$ \\
& $\mathbf{2}$ & $(4.4)$ & $(2.8)$ & $(1.4)$ & $(1.1)$ & $(1.0)$ \\
& $\mathbf{2 4}$ & $\mathbf{5 1}$ & $\mathbf{1 9}$ & $\mathbf{5}$ & $\mathbf{2}$ \\
& $\mathbf{3}$ & $(3.7)$ & $(3.8)$ & $(3.7)$ & $(2.2)$ & $(1.1)$ \\
& $\mathbf{6}$ & $\mathbf{6}$ & $\mathbf{5 1}$ & $\mathbf{2 0}$ & $\mathbf{3}$ \\
& $\mathbf{4}$ & $(2.3)$ & $(3.4)$ & $(3.9)$ & $(2.2)$ & $(1.5)$ \\
& $\mathbf{3}$ & $\mathbf{5}$ & $\mathbf{2 0}$ & $\mathbf{5 5}$ & $\mathbf{1 8}$ \\
& $\mathbf{5}$ & $(1.3)$ & $(2.0)$ & $(3.6)$ & $(3.3)$ & $(3.4)$ \\
& $\mathbf{1}$ & $\mathbf{1}$ & $\mathbf{4}$ & $\mathbf{1 7}$ & $\mathbf{7 6}$ \\
& $(1.0)$ & $(0.8)$ & $(1.8)$ & $(2.2)$ & $(3.3)$
\end{tabular}

Os resultados para os níveis de produtividade são bastante similares aos níveis da receita, embora com persistência das posições relativas ligeiramente menores - ou seja, mais valores diferentes de zero são encontrados fora da diagonal principal. No entanto, as maiores probabilidades ainda dominam a diagonal da matriz, indicando a alta persistência da posição relativa das firmas em termos de produtividade para todos os setores.

Dosi et al. (2012) e Mathew (2013) propõem um padrão de classificação das firmas de acordo com os movimentos apresentados na matriz de transição. A Tabela 4 apresenta este padrão. 
Tabela 4. Identificação de grupos de retardatários (A), gazelas (B), líderes (C) e ultrapassados (D) na MTP.

\begin{tabular}{ccccccc} 
& & $\mathrm{t}+1$ & & & & \\
\cline { 3 - 7 } $\mathrm{t}$ & 1 & 1 & 2 & 3 & 4 & 5 \\
\hline & 2 & $\mathrm{~A}$ & $\mathrm{~A}$ & & $\mathrm{~B}$ \\
& 3 & $\mathrm{~A}$ & $\mathrm{~A}$ & & $\mathrm{~B}$ \\
& 4 & & & & \\
& 5 & & & & $\mathrm{C}$ \\
\hline
\end{tabular}

Fonte: Elaboração própria com bases em Dosi (2012)

Tanto para os níveis de produtividade quanto para o tamanho das firmas observa-se uma grande parcela de retardatários e líderes nos mercados - neo-dualismo. Não ocorrem grandes mudanças de patamares para as receitas nem para cima e nem para baixo, o que faz com que o número de ultrapassados e de gazelas seja zero ou excepcionalmente $1 \%$. Para a produtividade as probabilidades de gazelas e ultrapassados é um pouco maior, mas ainda muito baixa para justificar que as firmas façam grandes saltos de produtividade como se poderia supor sob regimes de destruição criadora - como verificado pela literatura internacional no estudo de caso de outros países.

As matrizes de transição para as taxas de crescimento e variação da produtividade, também confirmam as evidências empíricas da literatura internacional, isto é, a ausência de persistência para as taxas de crescimento e variação da produtividade - ver Tabelas 4 e 5. MTs para período distintos da mesma base de dados são apresentadas no anexo.

Tabela 4. Matrizes de transição para a Variação da Receita Total. Medias e Desvios-padrão das amostras de 1996-2013.

10

\begin{tabular}{|c|c|c|c|c|c|}
\hline & \multicolumn{5}{|c|}{$t+1$} \\
\hline & 1 & 2 & 3 & 4 & 5 \\
\hline \multirow{2}{*}{1} & 23 & 18 & 15 & 17 & 27 \\
\hline & (3.3) & (2.0) & (2.1) & (2.5) & (3.8) \\
\hline \multirow{2}{*}{2} & 20 & 25 & 22 & 18 & 11 \\
\hline & (2.1) & (1.9) & (2.1) & (2.7) & (2.1) \\
\hline \multirow{2}{*}{3} & 17 & 25 & 26 & 20 & 10 \\
\hline & (2.7) & (1.9) & (2.1) & (2.8) & (2.2) \\
\hline \multirow{2}{*}{4} & 20 & 20 & 22 & 24 & 16 \\
\hline & (3.5) & (3.5) & (1.2) & (3.7) & (2.8) \\
\hline \multirow{2}{*}{5} & 20 & 13 & 14 & 21 & 38 \\
\hline & (1.8) & (1.4) & (2.2) & (1.7) & (4.7) \\
\hline
\end{tabular}

28

28
20

\begin{tabular}{l|ccccc}
\multicolumn{5}{c}{} & \multicolumn{5}{c}{$\mathbf{t + 1}$} \\
$\mathbf{1}$ & $\mathbf{1}$ & $\mathbf{2}$ & $\mathbf{3}$ & $\mathbf{4}$ & 5 \\
\cline { 2 - 6 } $\mathbf{2}$ & $\mathbf{2 3}$ & $\mathbf{1 8}$ & $\mathbf{1 6}$ & $\mathbf{1 8}$ & $\mathbf{2 4}$ \\
& $(3.6)$ & $(1.9)$ & $(2.5)$ & $(3.2)$ & $(5.9)$ \\
& $\mathbf{2 0}$ & $\mathbf{2 4}$ & $\mathbf{2 3}$ & $\mathbf{1 9}$ & $\mathbf{1 4}$ \\
$\mathbf{3}$ & $(2.3)$ & $(3.2)$ & $(2.7)$ & $(3.2)$ & $(3.6)$ \\
& $\mathbf{1 8}$ & $\mathbf{2 4}$ & $\mathbf{2 5}$ & $\mathbf{2 2}$ & $\mathbf{1 3}$ \\
$\mathbf{4}$ & $(2.4)$ & $(2.5)$ & $(2.9)$ & $(3.4)$ & $(2.4)$ \\
& $\mathbf{1 9}$ & $\mathbf{2 0}$ & $\mathbf{2 3}$ & $\mathbf{2 3}$ & $\mathbf{1 5}$ \\
$\mathbf{5}$ & $(3.0)$ & $(2.9)$ & $(3.2)$ & $(3.7)$ & $(4.4)$ \\
& $\mathbf{2 1}$ & $\mathbf{1 5}$ & $\mathbf{1 4}$ & $\mathbf{1 8}$ & $\mathbf{3 4}$ \\
& $(3.5)$ & $(2.0)$ & $(2.2)$ & $(2.8)$ & $(4.7)$
\end{tabular}

29

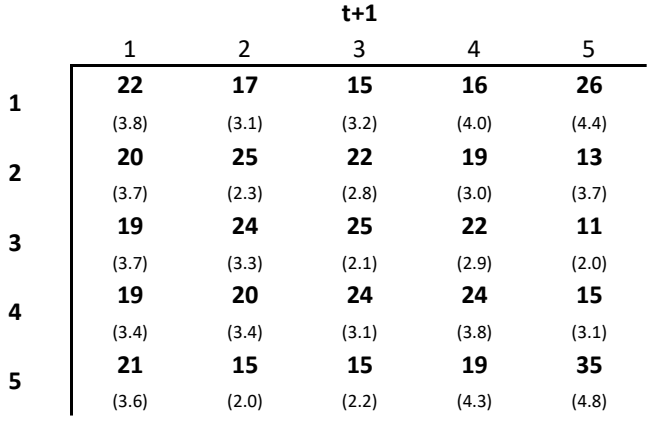


Tabela 5. Matrizes de transição para a Variação da Produtividade (VTI/ PO). Medias e Desvios-padrao das amostras de 1996-2013.

10

\begin{tabular}{|c|c|c|c|c|c|}
\hline \multirow[t]{2}{*}{10} & \multicolumn{5}{|c|}{$t+1$} \\
\hline & 1 & 2 & 3 & 4 & 5 \\
\hline \multirow{2}{*}{1} & 14 & 12 & 14 & 26 & 54 \\
\hline & (1.5) & (1.6) & (1.5) & $(2.0)$ & (4.5) \\
\hline \multirow{2}{*}{2} & 15 & 22 & 27 & 23 & 9 \\
\hline & (1.0) & (1.5) & (2.4) & (2.2) & (2.3) \\
\hline \multirow{2}{*}{3} & 17 & 27 & 27 & 18 & 6 \\
\hline & (1.6) & (2.3) & (1.4) & $(2.5)$ & (1.9) \\
\hline \multirow{2}{*}{4} & 27 & 25 & 19 & 15 & 7 \\
\hline & (2.2) & (1.3) & (1.3) & $(1.6)$ & (2.3) \\
\hline \multirow{2}{*}{5} & 26 & 14 & 13 & 18 & 24 \\
\hline & (2.5) & (1.8) & $(1.5)$ & (2.2) & $(4.2)$ \\
\hline
\end{tabular}

28

\begin{tabular}{cc|ccccc} 
& & 1 & 2 & 3 & 4 & 5 \\
\cline { 3 - 7 } $\mathbf{4}$ & $\mathbf{1 3}$ & $\mathbf{1 2}$ & $\mathbf{1 4}$ & $\mathbf{2 5}$ & $\mathbf{5 2}$ \\
& $\mathbf{2}$ & $(1.9)$ & $(1.7)$ & $(1.8)$ & $(3.0)$ & $(5.7)$ \\
& $\mathbf{1 4}$ & $\mathbf{2 1}$ & $\mathbf{2 7}$ & $\mathbf{2 5}$ & $\mathbf{1 0}$ \\
& $\mathbf{3}$ & $(1.5)$ & $(3.3)$ & $(1.7)$ & $(3.4)$ & $(3.8)$ \\
& $\mathbf{1 7}$ & $\mathbf{2 7}$ & $\mathbf{2 7}$ & $\mathbf{1 8}$ & $\mathbf{7}$ \\
& $\mathbf{4}$ & $(2.0)$ & $(2.7)$ & $(3.3)$ & $(2.2)$ & $(2.9)$ \\
& $\mathbf{2 7}$ & $\mathbf{2 5}$ & $\mathbf{1 9}$ & $\mathbf{1 5}$ & $\mathbf{8}$ \\
& $\mathbf{5}$ & $(3.0)$ & $(2.5)$ & $(1.8)$ & $(2.3)$ & $(3.2)$ \\
& $\mathbf{2 9}$ & $\mathbf{1 5}$ & $\mathbf{1 2}$ & $\mathbf{1 6}$ & $\mathbf{2 2}$ \\
& $(2.5)$ & $(2.5)$ & $(2.2)$ & $(2.5)$ & $(5.5)$
\end{tabular}

20

\begin{tabular}{|c|c|c|c|c|c|}
\hline & \multicolumn{5}{|c|}{$t+1$} \\
\hline & 1 & 2 & 3 & 4 & 5 \\
\hline \multirow{2}{*}{1} & 16 & 11 & 14 & 23 & 50 \\
\hline & (2.8) & (2.2) & (1.6) & $(4.0)$ & $(7.2)$ \\
\hline \multirow{2}{*}{2} & 15 & 22 & 26 & 24 & 12 \\
\hline & (2.8) & (2.8) & (3.2) & (2.2) & (4.4) \\
\hline \multirow{2}{*}{3} & 17 & 27 & 27 & 19 & 7 \\
\hline & (2.6) & (2.1) & (2.9) & (2.7) & (2.1) \\
\hline \multirow{2}{*}{4} & 26 & 24 & 21 & 17 & 9 \\
\hline & (3.3) & (2.1) & (2.5) & (2.5) & (3.2) \\
\hline \multirow{2}{*}{5} & 27 & 16 & 12 & 17 & 23 \\
\hline & (3.1) & (1.6) & (2.3) & (3.4) & (4.2) \\
\hline
\end{tabular}

29

\begin{tabular}{|c|c|c|c|c|c|}
\hline & 1 & 2 & 3 & 4 & 5 \\
\hline \multirow{2}{*}{1} & 14 & 11 & 13 & 24 & 49 \\
\hline & (1.9) & (2.3) & (2.4) & (3.8) & $(7.0)$ \\
\hline \multirow{2}{*}{2} & 14 & 21 & 28 & 24 & 11 \\
\hline & (2.3) & (3.1) & (2.8) & (3.7) & (3.3) \\
\hline \multirow{2}{*}{3} & 16 & 26 & 27 & 19 & 8 \\
\hline & (2.0) & (3.1) & (3.5) & (2.5) & (3.1) \\
\hline \multirow{2}{*}{4} & 26 & 26 & 20 & 17 & 8 \\
\hline & (3.1) & (2.6) & (2.8) & (3.1) & (3.2) \\
\hline \multirow{2}{*}{5} & 30 & 15 & 12 & 16 & 24 \\
\hline & (3.4) & (2.6) & (2.4) & (3.3) & (5.4) \\
\hline
\end{tabular}

Note que as probabilidades de transição são mais distribuídas nas diferentes posições das MTs se comparadas às probabilidades observadas para os níveis de receita e produtividade, indicando a ausência de persistência nas taxas de crescimento e de ganhos de produtividade das firmas para todos os setores. Em outras palavras, as firmas dificilmente se mantem no mesmo quintil de crescimento - seja das receitas ou da produtividade - por longos períodos de tempo.

Em suma, as MTs confirmam, empiricamente, a persistência das posições relativas e não persistências das taxas de crescimento para todos os setores analisados. Obviamente, os dois fatos estilizados se retroalimentam, visto que se as taxas de crescimento não são persistentes no tempo e se dão de maneira mais aleatória e distribuída, a posição relativa das firmas não deve se alterar.

\section{Considerações finais}

Este artigo buscou verificar a ocorrência de alguns fatos estilizados comumente encontrados pela literatura internacional em outros países para o Brasil. Mais especificamente, utilizamos métodos não paramétricos para confirmar a ocorrência de expressiva heterogeneidade das firmas, assimetria nas distribuições, persistência das suas posições relativas no tempo e nãopersistência das taxas de crescimento. Dado o nosso conhecimento, o estudo é inédito para o caso brasileiro, de maneira que contribui não só para o melhor entendimento das estruturas industriais nacionais e seu comportamento no tempo, mas também com a robustez dos resultados encontrados de forma empírica para outros países e recortes temporais.

Para os setores selecionados e o total da manufatura, verificou-se a persistência de expressiva heterogeneidade das firmas e assimetria positiva das distribuições, bem como alta probabilidade de permanência nas posições relativas de desempenho. Em outras palavras, os 
mercados acomodam firmas heterogêneas, com elevada proporção de firmas menores ou de baixa produtividade, ao mesmo tempo em que as firmas tendem a manter sua posição no ranking de produtividade e tamanho ao longo do tempo. Por outro lado, a mesma persistência não se verifica quando analisamos o crescimento das firmas e seus ganhos de produtividade. Estes fatos estilizados em conjunto desafiam a crença exacerbada na eficiência do mercado como mecanismo de seleção, a ocorrência de eventos de destruição criadora e as possibilidades de catching-up das firmas nos diferentes setores.

\section{Agradecimentos}

Os autores agradecem ao Conselho Nacional de Desenvolvimento Científico e Tecnológico - CNPq, processo 444183/2015-9; à Fundação de Amparo à Pesquisa do Estado de São Paulo Fapesp, processo 2015/17059-3 e à Fundo de Apoio ao Ensino, à Pesquisa e a Extensão - Faepex, processo 27316 pelo suporte financeiro. Os autores também agradecem aos técnicos do setor de indústria do IBGE e aos colegas do IPEA pelas suas valiosas contribuições para o desenvolvimento da pesquisa.

\section{Referências}

BALDWIN, J. R.; RAFIQUZZAMAN, M. Selection versus evolutionary adaptation: Learning and post-entry performance. International Journal of Industrial Organization, v. 13, n. 4, p. 501-522, dez. 1995.

BARTELSMAN, E. J.; DOMS, M. Understanding Productivity: Lessons from Longitudinal Microdata. Journal of Economic Literature, v. 38, n. 3, p. 569-594, 2000.

BOLDRINI, J. L. Álgebra Linear. São Paulo: Harper \& Row do Brasil, 1986.

BOTTAZZI, G. et al. Invariances and Diversities in the Patterns of Industrial Evolution: Some Evidence from Italian Manufacturing Industries. Small Business Economics, v. 29, n. 1-2, p. 137-159, 1 maio 2007.

BOTTAZZI, G. et al. Corporate growth and industrial dynamics: evidence from French manufacturing. Applied Economics, v. 43, n. 1, p. 103-116, jan. 2011.

Corporate growth and industrial structures: some evidence from the Italian manufacturing industry. Industrial and Corporate Change, v. 11, n. 4, p. 705-723, 1 ago. $2002 \mathrm{~b}$.

CATELA, E. Y.; CIMOLI, M.; PORCILE, G. Productivity and Structural Heterogeneity in the Brazilian Manufacturing Sector: Trends and Determinants. Oxford Development Studies, v. 43, n. 2, p. 232-252, 2015.

CEFIS, E. Is there persistence in innovative activities? International Journal of Industrial Organization, v. 21, n. 4, p. 489-515, 2003.

COAD, A.; SEGARRA, A.; TERUEL, M. Like milk or wine: Does firm performance improve with age? Structural Change and Economic Dynamics, v. 24, n. 1, p. 173-189, 2013.

DOSI, G. Statistical regularities in the evolution of industries: A guide through some evidence and challenges for the theory. 2005. 
. Technological Revolutions and the Evolution of Industrial Structures: Assessing the Impact of New Technologies upon the Size and Boundaries of Firms. Capitalism and Society, v. 3, n. 1, p. 10-67, 2008.

. Turbulence underneath the big calm? The micro-evidence behind Italian productivity dynamics. Small Business Economics, v. 39, n. 4, p. 1043-1067, 2012.

Productivity, market selection, and corporate growth: comparative evidence across US and Europe. Small Business Economics, 2015.

DOSI, G.; NELSON, R. R. Technical change and industrial dynamics as evolutionary processes. In: HALL, B. H.; ROSENBERG, N. (Eds.). . Handbook of the Economics of Innovation. 1. ed. [s.1.] Academic Press, 2010. p. 51-128.

DOSI, G.; PEREIRA, M. C.; VIRGILLITO, M. E. The footprint of evolutionary processes of learning and selection upon the statistical properties of industrial dynamics. Industrial and Corporate Change, p. dtw044, 25 dez. 2016.

DUNNE, T.; ROBERTS, M. J.; SAMUELSON, L. Patterns of Firm Entry and Exit in U.S. Manufacturing Industries. The RAND Journal of Economics, v. 19, n. 4, p. 495, 1988.

ERIC J. BARTELSMAN, P. J. D. Productivity Dynamics: U.S. Manufacturing Plants, 1972-1986. Journal of Productivity Analysis, n. 9, p. 5-34, 1998.

FOSTER, L.; HALTIWANGER, J.; SYVERSON, C. Reallocation, Firm Turnover, and Efficiency:Selection on Productivity or Profitability? American Economic Review, n. 2000, p. 394-425, 2008.

GEROSKI, P. A. What do we know about entry? International Journal of Industrial Organization, v. 13, n. 4, p. 421-440, dez. 1995.

MATHEW, N. Drivers of Firm Growth : Micro-evidence from Indian Manufacturing. p. $1-28,2013$.

Drivers of firm growth: micro-evidence from Indian manufacturing. Journal of Evolutionary Economics, p. 1-27, mar. 2017.

MESSA, ALEXANDRE; ROMMINGER, ALFREDO ERIC; AURICCHIO LEDO, B. et al. Produtividade no Brasil: Desempenho e Determinantes. Brasília: IPEA/ABDI, 2015.

METCALFE, J. S. Competition, Fisher's Principle and increasing returns in the selection process. Journal of Evolutionary Economics, v. 4, n. 4, p. 327-346, dez. 1994.

NEGRI, F. DE; RICARDO CAVALCANTE, L. Evolução Recente dos Indicadores de Produtividade no Brasil. Radar/Diset, p. 7-15, ago. 2013.

NELSON, R. R.; WINTER, S. G. An evolutionary theory of economic change. [s.1.] Belknap Press of Harvard University Press, 1982.

SEBRAE. Participação das Micro e Pequenas Empresas na Economia Brasileira. [s.l: s.n.]. Disponível em: <https://www.sebrae.com.br/Sebrae/Portal Sebrae/Estudos e Pesquisas/Participacao das micro e pequenas empresas.pdf>. Acesso em: 20 abr. 2017.

SILVERMAN, B. W. Density estimation for statistics and data analysis. [s.1.] Chapman and Hall, 1986. 
SYVERSON, C. What Determines Productivity? Journal of Economic Literature, v. 49, n. 2, p. 326-365, jun. 2011.

TRIGUERO, Á.; CÓRCOLES, D. Understanding innovation: An analysis of persistence for Spanish manufacturing firms. Research Policy, v. 42, p. 340-352, 2013. 


\section{Apêndice}

Tabela 6. Matrizes de transição para taxas de crescimento das firmas para os quatro setores selecionados e três períodos específicos.

10

\begin{tabular}{|c|c|c|c|c|c|}
\hline \multirow[t]{2}{*}{10} & \multicolumn{5}{|c|}{1997} \\
\hline & 1 & 2 & 3 & 4 & 5 \\
\hline 1 & 86 & 8 & 0 & - & - \\
\hline 2 & 14 & 78 & 9 & 0 & 0 \\
\hline 1996 & - & 13 & 81 & 9 & 1 \\
\hline 4 & - & 1 & 9 & 85 & 10 \\
\hline 5 & - & 0 & 1 & 5 & 89 \\
\hline
\end{tabular}

10

\begin{tabular}{|c|c|c|c|c|c|}
\hline & & & & & \\
\hline & 1 & 2 & 3 & 4 & 5 \\
\hline 1 & 90 & 19 & 1 & 0 & - \\
\hline 2 & 10 & 77 & 13 & 0 & - \\
\hline 3 & 1 & 4 & 79 & 16 & 1 \\
\hline 4 & - & 0 & 7 & 80 & 10 \\
\hline 5 & - & - & 0 & 3 & 90 \\
\hline
\end{tabular}

20

\begin{tabular}{rrrrrr}
\multicolumn{7}{c}{1997} & & \\
1 & 1 & 2 & 3 & 4 & 5 \\
\hline 2 & 89 & 9 & 1 & - & - \\
3 & 11 & 80 & 16 & - & - \\
4 & - & 10 & 76 & 13 & - \\
5 & - & 1 & 5 & 83 & 10 \\
& - & - & 1 & 4 & 90
\end{tabular}

20

\begin{tabular}{|c|c|c|c|c|c|}
\hline \multirow{2}{*}{20} & \multicolumn{5}{|c|}{2012} \\
\hline & 1 & 2 & 3 & 4 & 5 \\
\hline 1 & 94 & 16 & - & - & - \\
\hline 2 & 6 & 77 & 19 & 0 & - \\
\hline 2011 & 1 & 7 & 75 & 16 & 0 \\
\hline 4 & - & 0 & 5 & 81 & 7 \\
\hline 5 & - & - & 0 & 3 & 93 \\
\hline
\end{tabular}

28

\begin{tabular}{|c|c|c|c|c|c|}
\hline \multirow{3}{*}{ (1) } & \multicolumn{5}{|c|}{1997} \\
\hline & \multirow{2}{*}{$\begin{array}{r}1 \\
78\end{array}$} & \multirow{2}{*}{$\frac{2}{14}$} & \multirow{2}{*}{$\begin{array}{l}3 \\
1\end{array}$} & \multirow{2}{*}{$\frac{4}{0}$} & \multirow{2}{*}{$\frac{5}{-}$} \\
\hline & & & & & \\
\hline \multirow{4}{*}{996} & 20 & 65 & 17 & \multirow{2}{*}{$\begin{array}{r}1 \\
14\end{array}$} & - \\
\hline & 0 & 20 & 64 & & 0 \\
\hline & 1 & 1 & 18 & 75 & 6 \\
\hline & - & 0 & 0 & 9 & 93 \\
\hline
\end{tabular}

28

\begin{tabular}{rrrrrr}
\multicolumn{7}{c}{} & \multicolumn{7}{c}{2012} & & \\
1 & 1 & 2 & 3 & 4 & 5 \\
\hline 2 & 85 & 14 & 1 & - & - \\
3 & 12 & 72 & 18 & 1 & - \\
4 & 2 & 12 & 69 & 19 & 0 \\
5 & 1 & 1 & 12 & 72 & 12 \\
& - & - & - & 7 & 87
\end{tabular}

29

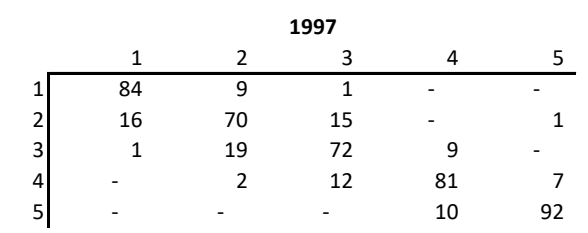

29

\begin{tabular}{rrrrrr}
\multicolumn{7}{c}{} & \multicolumn{7}{c}{2012} & & \\
1 & 1 & 2 & 3 & 4 & 5 \\
\hline 2 & 79 & 20 & - & - & - \\
3 & 20 & 69 & 11 & - & - \\
4 & 1 & 10 & 79 & 13 & 0 \\
5 & - & 1 & 11 & 83 & 7 \\
& - & - & - & 4 & 92
\end{tabular}

10

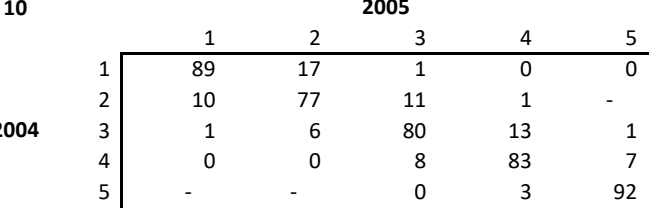

20

\begin{tabular}{rrrrrr}
\multicolumn{7}{c}{} & \multicolumn{2}{c}{2005} & & \\
\multicolumn{1}{c}{1} & 1 & 2 & 3 & 4 & 5 \\
\cline { 2 - 6 } 2 & 92 & 7 & 0 & - & - \\
3 & 7 & 87 & 14 & 0 & - \\
4 & 1 & 6 & 77 & 17 & 0 \\
5 & - & - & 9 & 79 & 7 \\
& - & - & 0 & 3 & 92
\end{tabular}

28

\begin{tabular}{r|rrrrr}
\multicolumn{7}{c}{} & \multicolumn{7}{c}{2005} \\
1 & 1 & 2 & 3 & 4 & 5 \\
\cline { 2 - 7 } 2 & 91 & 13 & 0 & - & - \\
3 & 8 & 70 & 22 & 2 & - \\
4 & 1 & 17 & 65 & 20 & 0 \\
5 & 0 & - & 12 & 70 & 12 \\
& - & - & 0 & 9 & 88
\end{tabular}

29

2005

2004

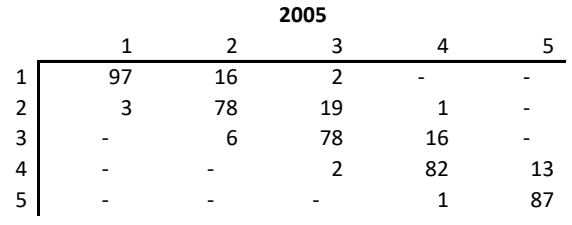


Tabela 7. Matrizes de transição para taxas de crescimento das firmas para os quatro setores selecionados e três períodos específicos.

\begin{tabular}{|c|c|c|c|c|c|c|c|c|c|c|c|c|c|}
\hline 10 & & \multicolumn{4}{|c|}{ 1997-1998 } & \multicolumn{3}{|c|}{10} & \multicolumn{4}{|c|}{$2005-2006$} & \\
\hline & 2 & 20 & 25 & 25 & 17 & 10 & & 2 & 20 & 26 & 20 & 20 & 8 \\
\hline & 4 & 23 & 17 & 21 & 25 & 20 & & 4 & 17 & 18 & 24 & 25 & 20 \\
\hline & 5 & 19 & 15 & 14 & 19 & 26 & & 5 & 19 & 13 & 13 & 21 & 37 \\
\hline
\end{tabular}

10

2012-2013

\begin{tabular}{rrrrrr}
\multicolumn{1}{c}{} & 1 & 2 & 3 & 4 & 5 \\
\cline { 2 - 6 } 2 & 24 & 19 & 15 & 15 & 24 \\
3 & 20 & 27 & 24 & 18 & 12 \\
4 & 16 & 24 & 28 & 24 & 8 \\
5 & 23 & 19 & 23 & 22 & 15 \\
& 17 & 10 & 11 & 20 & 41
\end{tabular}

\begin{tabular}{|c|c|c|c|c|}
\hline & & 1998 & & \\
\hline 1 & 2 & 3 & 4 & 5 \\
\hline 27 & 17 & 16 & 18 & 19 \\
\hline 20 & 28 & 26 & 12 & 11 \\
\hline 19 & 21 & 24 & 25 & 14 \\
\hline 19 & 18 & 20 & 28 & 13 \\
\hline 15 & 16 & 15 & 17 & 43 \\
\hline
\end{tabular}

20

2011-2012

\begin{tabular}{rrrrrr}
\multicolumn{1}{c}{} & 1 & 2 & 3 & 4 & 5 \\
\cline { 2 - 6 } 1 & 20 & 17 & 17 & 19 & 23 \\
2 & 21 & 25 & 25 & 18 & 11 \\
3 & 14 & 24 & 28 & 26 & 12 \\
4 & 21 & 22 & 18 & 20 & 21 \\
5 & 24 & 11 & 11 & 16 & 34
\end{tabular}

28

\begin{tabular}{rrrrrr}
\multicolumn{7}{c}{$1997-1998$} \\
1 & 2 & 3 & 4 & 5 \\
\cline { 2 - 6 } 2 & 18 & 14 & 17 & 28 \\
4 & 21 & 18 & 23 & 21 & 15 \\
& 16 & 24 & 29 & 21 & 12 \\
21 & 18 & 20 & 23 & 21 \\
& 21 & 23 & 23 & 18 & 25
\end{tabular}

28

2011-2012

\begin{tabular}{rrrrrr}
\multicolumn{7}{c}{} & \multicolumn{7}{c}{$\mathbf{2 0 1 2 - 2 0 1 3}$} \\
1 \\
\cline { 2 - 6 } 2 & 20 & 13 & 15 & 21 & 35 \\
3 & 20 & 25 & 25 & 19 & 12 \\
4 & 21 & 23 & 26 & 22 & 12 \\
5 & 19 & 25 & 20 & 24 & 14 \\
& 21 & 14 & 14 & 15 & 27
\end{tabular}

29

\begin{tabular}{rrrrrr}
\multicolumn{7}{c}{} & \multicolumn{1}{c}{$\mathbf{1 9 9 7 - 1 9 9 8}$} \\
1 & 1 & 2 & 3 & 4 & 5 \\
\cline { 2 - 6 } 2 & 23 & 15 & 13 & 12 & 26 \\
3 & 21 & 26 & 23 & 17 & 15 \\
4 & 16 & 21 & 26 & 26 & 12 \\
5 & 20 & 21 & 23 & 27 & 11 \\
& 20 & 17 & 15 & 17 & 36
\end{tabular}

29

\begin{tabular}{rrrrrr}
\multicolumn{7}{c}{} & \multicolumn{2}{c}{$\mathbf{2 0 1 2 - 2 0 1 3}$} \\
1 \\
\cline { 2 - 5 } & 1 & 2 & 3 & 4 & 5 \\
\hline 3 & 21 & 11 & 15 & 25 & 29 \\
4 & 19 & 23 & 19 & 20 & 19 \\
5 & 22 & 27 & 28 & 18 & 9 \\
& 15 & 27 & 26 & 20 & 11 \\
& 24 & 13 & 12 & 16 & 31
\end{tabular}

29

\begin{tabular}{rrrrrrr}
\multicolumn{2}{l}{29} & \multicolumn{6}{c}{$\mathbf{2 0 0 5 - 2 0 0 6}$} \\
\cline { 3 - 7 } $2004-2005$ & 1 & 2 & 3 & 4 & 5 \\
\hline & 2 & 24 & 17 & 20 & 14 & 22 \\
& 3 & 15 & 24 & 25 & 21 & 16 \\
& 4 & 18 & 22 & 26 & 22 & 13 \\
& 5 & 21 & 22 & 18 & 24 & 18 \\
& & 22 & 16 & 12 & 18 & 32
\end{tabular}

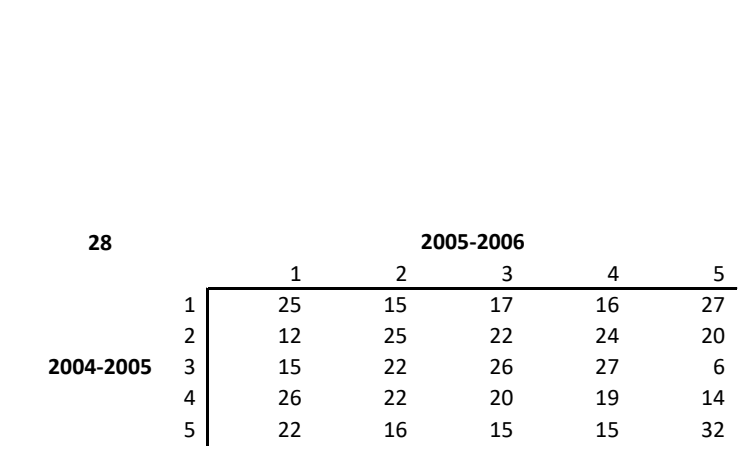

\begin{tabular}{lr|rrrrr}
2 & \multicolumn{6}{c}{ 2005-2006 } \\
\cline { 3 - 7 } $2004-2005$ & 1 & 2 & 3 & 4 & 5 \\
\hline & 2 & 23 & 20 & 10 & 20 & 18 \\
& 3 & 21 & 29 & 22 & 15 & 14 \\
& 4 & 17 & 25 & 27 & 19 & 14 \\
& 5 & 20 & 13 & 26 & 28 & 13 \\
& & 20 & 13 & 15 & 18 & 40
\end{tabular}

(1)

